\title{
Badges for sharing data and code at Biostatistics: an
}

\section{observational study [version 1; peer review: 1 approved, 1}

\section{approved with reservations]}

\author{
Anisa Rowhani-Farid (D), Adrian G. Barnett (iD) \\ Institute of Health and Biomedical Innovation, Queensland University of Technology, Brisbane, Queensland, 4001, Australia
}

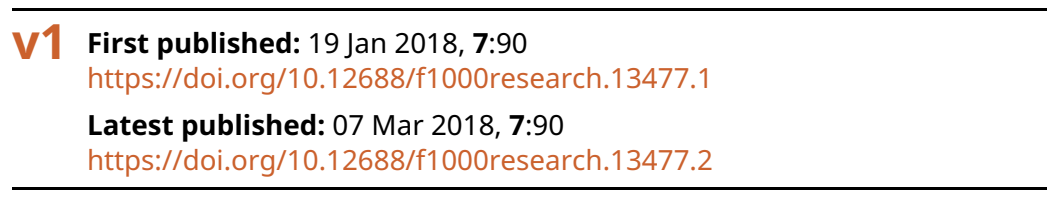

\section{Abstract}

Background: Reproducible research includes sharing data and code. The reproducibility policy at the journal Biostatistics rewards articles with badges for data and code sharing. This study investigates the effect of badges at increasing reproducible research, specifically, data and code sharing, at Biostatistics.

Methods: The setting of this observational study is the Biostatistics and Statistics in Medicine (control journal) online research archives. The data consisted of 240 randomly sampled articles from 2006 to 2013 (30 articles per year) per journal, a total sample of 480 articles. Data analyses included: plotting probability of data and code sharing by article submission date, and Bayesian logistic regression modelling to test for a difference in the probability of making data and code available after the introduction of badges at Biostatistics.

Results: The probability of data sharing was higher at Biostatistics than the control journal but the probability of code sharing was comparable for both journals. The probability of data sharing increased by 3.5 times (95\% credible interval: 1.4 to 7.4 times, p-value probability that sharing increased: 0.996) after badges were introduced at Biostatistics. On an absolute scale, however, this difference was only a $7.3 \%$ increase in data sharing (95\% CI: 2 to 14\%, p-value: 0.996). Badges did not have an impact on code sharing at the journal (mean increase: 1.1 times, 95\% credible interval: 0.45 to 2.14 times, p-value probability that sharing increased: 0.549).

Conclusions: The effect of badges at Biostatistics was a $7.3 \%$ increase in the data sharing rate, 5 times less than the effect of badges on data sharing at Psychological Science (37.9\% badge effect). Though the effect of badges at Biostatistics did not impact code sharing, and was associated with only a moderate effect on data sharing, badges are an interesting step that journals are taking to incentivise and promote reproducible research.

\section{Open Peer Review \\ Approval Status \\ 1 \\ 2 \\ version 2 \\ (revision) \\ 07 Mar 2018 \\ version 1 \\ 19 Jan 2018

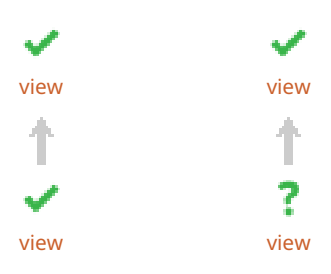 \\ 1. Gustav Nilsonne ID, Stockholm University, \\ Stockholm, Sweden \\ Karolinska Institutet, Stockholm, Sweden \\ 2. Bastian Greshake ID, Lawrence Berkeley \\ National Laboratory, Berkeley, USA \\ Open Humans (openhumans.org), Frankfurt am Main, Germany}

Any reports and responses or comments on the article can be found at the end of the article. 


\section{Keywords}

Reproducibility, incentives, rewards, data sharing, code sharing, meta-

research

This article is included in the Research on

Research, Policy \& Culture gateway.

Corresponding author: Anisa Rowhani-Farid (anisa.rowhanifarid@hdr.qut.edu.au)

Author roles: Rowhani-Farid A: Conceptualization, Investigation, Methodology, Writing - Original Draft Preparation; Barnett AG:

Conceptualization, Data Curation, Formal Analysis, Methodology, Software, Supervision, Validation, Visualization, Writing - Review \&

Editing

Competing interests: No competing interests were disclosed.

Grant information: This study was supported in kind by the Institute of Health and Biomedical Innovation at the Queensland University of Technology.

The funders had no role in study design, data collection and analysis, decision to publish, or preparation of the manuscript.

Copyright: @ 2018 Rowhani-Farid A and Barnett AG. This is an open access article distributed under the terms of the Creative Commons Attribution License, which permits unrestricted use, distribution, and reproduction in any medium, provided the original work is properly cited.

How to cite this article: Rowhani-Farid A and Barnett AG. Badges for sharing data and code at Biostatistics: an observational study [version 1; peer review: 1 approved, 1 approved with reservations] F1000Research 2018, 7:90

https://doi.org/10.12688/f1000research.13477.1

First published: 19 Jan 2018, 7:90 https://doi.org/10.12688/f1000research.13477.1 


\section{Introduction}

Historically, the replication of a scientific experiment has been the measure of its validity, however, not all experiments can be replicated in their totality ${ }^{1}$. 'Replicability' is the ability of a researcher to duplicate the results of a prior study if the same procedures are followed but new data are collected ${ }^{2}$. In 2009, Roger Peng mentioned in an editorial in Biostatistics that the minimum standard that could bridge the gap between replicability and nothing is "reproducible research"1. 'Reproducibility' is the ability of a researcher to duplicate the results of a prior study using the same materials as were used by the original investigator ${ }^{2}$. Reproducibility was defined by Peng in terms of sharing the data and computer code used to analyse the data and he described it as the "cornerstone of the scientific method". In a perspective piece in 2011, Peng likened reproducibility to a spectrum, at one end being the gold standard of full replication, and at the other, publication only ${ }^{3}$. Given the expectation that data will be accessible, researchers who refuse to share the evidentiary basis behind their conclusions, or the materials needed to reproduce published experiments, fail to maintain the standards of science $^{4}$. Although in some instances highly-sensitive data cannot be shared for legal or privacy reasons.

Scientific journals are critical to changing the culture of research. Many journals are introducing data sharing policies, but studies have shown that policies alone are not effective in promoting a culture of sharing and that scientists potentially need to be rewarded for good behaviour ${ }^{5}$. Ioannidis et al. discuss changing the reward criteria to include 'reproducible' and 'sharing' using the PQRST criteria - productive, high-quality, reproducible, shareable, and translatable ${ }^{6}$. A systematic review of incentives that motivated researchers to share their data in the health and medical research community, uncovered only one evidence-based incentive that increased data sharing at the journal Psychological Science from 1.5\% pre-incentive (2012) to $39.4 \%$ post-incentive $(2015)^{7,8}$. This incentive was an open data badge developed by the Center of Open Science (COS) and introduced at the journal in January $2014^{8}$.

Badges for reproducible research were not an innovative creation of COS however. The journal Biostatistics introduced badges, or what they called kitemarks (named after the UK kitemark system of establishing product safety), on 1 July 2009 as part of their policy to reward reproducible research ${ }^{1}$. The policy was introduced by Roger Peng, the then Associate Editor for reproducibility $(\mathrm{AER})^{1}$. Sharing was not enforced, rather authors were encouraged to consider the reproducibility of their research ${ }^{1}$. From here on, kitemarks will be referred to as badges, using common terminology.

The reproducibility policy at the journal instructed authors to indicate in their submission if they intend to submit supplementary materials that include data, code, or both ${ }^{1}$. The policy rewarded articles with data available with the letter $\mathbf{D}$ on the front page of the published article PDF, articles with code available with a $\mathbf{C}$, and articles with data and code available and which were tested for reproducibility by the AER an $\mathbf{R}$ for reproducibility $^{1}$. It is important to note that data refers to raw data and not simulated data, which are commonly used in statistics.

The policy change at Biostatistics provided an ideal opportunity to replicate the findings of the Kidwell et al. badge study by examining sharing rates at another journal that offered a reward or incentive for reproducible research ${ }^{8}$. We note that Kidwell et al. examined data and material sharing only, as badges were not offered for code.

A survey conducted by Nature in 2016 indicates that the scientific community is in the midst of a reproducibility crisis? The current culture in science provides strong incentives for innovation and relatively weak incentives for certainty and reproducibility ${ }^{10}$. Within the current 'post-truth' era there is much public scrutiny and suspicion around the validity of science. Such a debate, compounded by the reproducibility crisis, signals a time for a cultural shift in the scientific research process ${ }^{11}$. The sharing of data, as well as the computer code used to analyse the data, should, where possible, be integral components of the research process, however data sharing rates have been as low as $0 \%{ }^{12}$. Of course, not all data can be shared due to legal and ethical constraints, but these are neither the only, nor main reasons behind low sharing rates ${ }^{13}$. Scientists are still exploring the barriers towards sharing and a key concern is that researchers are not incentivised to share ${ }^{3}$.

Aim

Our aim is to investigate the effect of badges at increasing reproducible research, specifically, data and code sharing, at Biostatistics.

\section{Methods}

\section{Participants}

This is an observational study with two journals, intervention and control, using a pre-post study design, with 30 randomly selected papers per year from 2006 to 2013 for each journal. We chose Statistics in Medicine as the control journal as it did not have a badges or any type of reproducible research reward scheme during those years, but is in the same field of research with similar goals of publishing papers on statistical methods development in health and medicine. The study setting is the Biostatistics and Statistics in Medicine research archive. All the information required was publicly available online, as such participant consent was not required and an ethics exemption (exemption number: 1700001051) was granted by the Office of Research Ethics and Integrity at the Queensland University of Technology.

\section{Sample size calculation and power}

A sample of only 19 papers per journal would have given us a $90 \%$ power to detect a difference in data sharing of $37.9 \%$, based on the effect of badges from the Kidwell et al. study ${ }^{8}$. This uses a two-sided 5\% significance level. We felt this sample was unrealistically small, hence we instead based our sample size on the practical considerations of reading papers and examining their data and code sharing choices, given the time constraints of the first author's (ARF) PhD. Thirty papers per year from 
2006 to 2013 for two journals is a total sample of 480 papers, which is practically possible, and provides good coverage over the time of the policy change at Biostatistics.

\section{Data collection}

For each year and journal a random number generator was used to select the research articles (in Microsoft Excel 20161). Articles were included if they:

- Generated and analysed original data (article had data and code to share), or

- Conducted secondary analyses on a pre-existing dataset from another study (article had data and code to share), or

- Generated simulated data (article did not have data to share but had code to share)

Articles were excluded if:

- They were meta-analyses, meta-regressions, or systematic reviews, as these papers usually contain the data within the paper

- They were case series, opinion pieces or some other publication type without data or code

If an article was excluded then we sampled another article from the same year and journal to maintain the sample size. ARF read the research papers and extracted the details of the articles included in the study. Each article was screened using these search terms: "data", "code", "package", "available", "https", "www", "figshare", and "github". For the included articles, the following variables were documented: submission date, data sharing statement, data availability, hyperlink to dataset, code sharing statement, code availability, hyperlink to code, and badge allocation (for Biostatistics articles).

The second author (AGB) independently assessed data and code sharing for 20 randomly selected articles. There were minor discrepancies between the authors, which were resolved by discussion.

Using definitions from our previous work $^{5}$, each research article was categorised for data and code sharing as:

\section{Data sharing}

available: articles that had a functioning link to a publicly available dataset deposited at a third-party site or attached as supplementary material to the electronic version of the article

potentially available: articles that indicated that the dataset was potentially available upon request from the authors

not available: articles that did not indicate the availability of the dataset analysed in the article or where the link to the data was no longer working

none to share: articles that used simulated data and so did not have a raw dataset to share
Code sharing

available: articles that had a functioning link to publicly available code deposited at a third-party site, or attached as supplementary material to the electronic version of the article or available within the article itself

potentially available: articles that indicated that the code was potentially available upon request from the authors

not available: articles that did not indicate the availability of the code used to analyse the data (raw or simulated) or where the link to the code was no longer working

\section{Intervention period}

We defined the intervention period based on the policy change date at Biostatistics and using the article's submission date as this is when authors are thinking about the journal requirements and perhaps becoming aware of the badge. Since the policy change was on 1 July 2009, papers submitted to Biostatistics after that date were in the intervention period. We included a six month gap before the policy change as an interim phase because papers submitted during this time (1 January 2009 to 1 July 2009) could experience the badge policy upon re-submission, so papers submitted in this period were categorized into the interim period. Any papers submitted to Biostatistics before 1 January 2009 were in the control period and all papers submitted to Statistics in Medicine were controls.

The first analysis examined data and code availability and probability of sharing over time using submission date. As a sensitivity analysis, we used the articles' publication dates extracted from PubMed in place of submission date. We conducted this sensitivity analysis to examine whether the policy was associated with a change based on the very latest date that authors could make changes to their papers.

\section{Statistics methods}

We plotted the binary data and code sharing over time and included a smooth curve to estimate the mean sharing rate over time in each journal. The smooth curves were made using a LOESS smooth with a span of 0.9 , and we also plotted the $95 \%$ confidence intervals. Papers where there was no data to share (i.e., using simulated data) were excluded from these plots.

To test for a difference in the probability of making data and code available after the introduction of badges, we used logistic regression and presented the results as prevalence ratios rather than odds ratios, as prevalence ratios are generally easier to understand $d^{14}$. Due to possible convergence issues with a standard logistic regression model using a log-link to estimate prevalence ratios, we ran a Bayesian logistic regression model using WinBUGS (version 1.3.4). Using a Bayesian model has the added advantage of giving $95 \%$ credible intervals and Bayesian p-values that are far easier to interpret than frequentist confidence intervals and p-values. The Bayesian p-values used here estimate the probability that sharing increased after the policy change at Biostatistics. As well as showing the change in data and code sharing probability, on the relative scale, of 
the prevalence ratio, we also show the absolute increase in sharing probability after the policy change together with $95 \%$ credible intervals.

In a sensitivity analysis we used a strong control for time by including year as a random effect, assuming that each year has its own data sharing rate. This essentially matches papers from Biostatistics and Statistics in Medicine from the same year. We did this to adjust for other changes over time, for example a potential increase over time in data and code depositories such as GitHub, Figshare, and Dryad, and a potential decrease in data and code availability for papers published many years ago because of broken links ${ }^{15}$.

The data analysis was made using the statistical software $\mathrm{R}$ (version 3.2.3).

\section{Results}

Frequency of data and code availability

Flow charts show the frequency of data and code availability for each journal (Figures $1 \mathrm{a}$ and 1b). Biostatistics had 8 articles with no data to share, bringing the sample with possible data available to $232 ; 20$ of which had data available, 3 had data potentially available and 209 had no data available. Statistics in Medicine had 31 articles with no data to share, bringing the sample with possible data available to $209 ; 3$ of which had data available, 4 had data potentially available and 202 had no data available.

The frequency of code availability for each journal is in Figures $2 \mathrm{a}$ and $2 \mathrm{~b}$, which were comparable for the two journals. Statistics in Medicine had 24 articles with code available, 27 potentially available, and 189 with no code available, while Biostatistics had 14 articles with code available, 22 potentially available, and 204 with no code available.

The data available and probability of sharing by submission date together with a smooth curve and $95 \%$ confidence intervals are in Figure 3a. The vertical red lines are at 1 July 2009, the date badges were introduced at Biostatistics, and 1 January 2009, six months prior to the policy change (interim period). It is clear that data availability and probability of sharing were greater over time in Biostatistics than in the control journal, Statistics in Medicine, but the probability of sharing data at Biostatistics was still low, at well below 0.25. Interestingly an increase in data sharing at Biostatistics took place before badges were introduced at the journal. The results of the sensitivity analysis using publication date are shown in Figure $3 b$. The smooth curves a

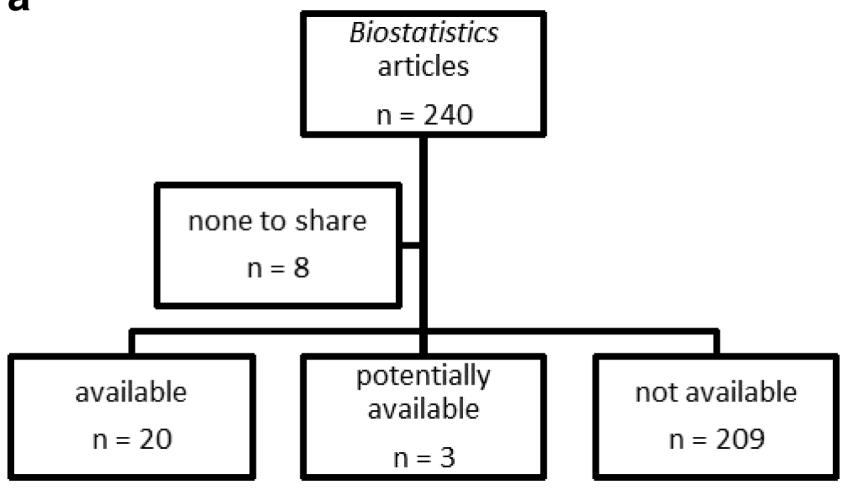

b

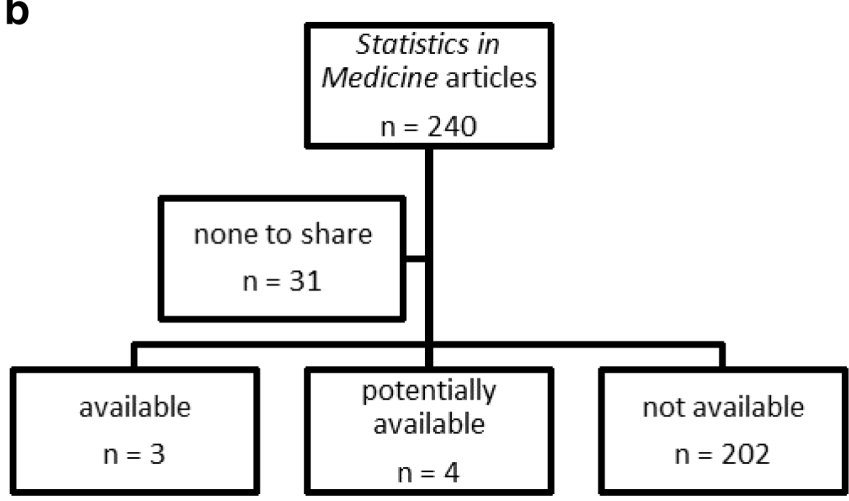

Figure 1. a: Flow chart of data availability. Randomly selected Biostatistics articles from 2006 to 2013, b: Flow charts of data availability Randomly selected Statistics in Medicine articles from 2006 to 2013.

a

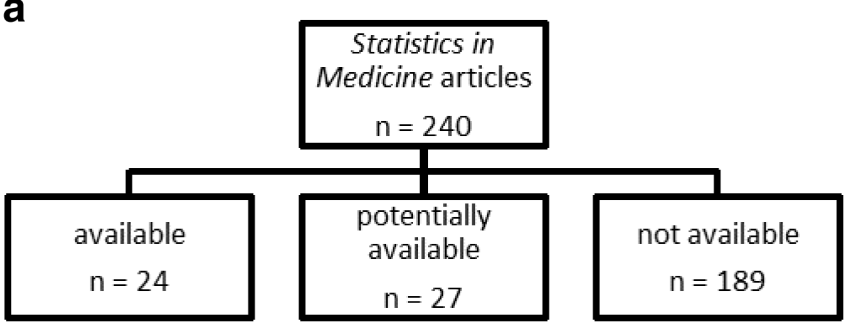

b

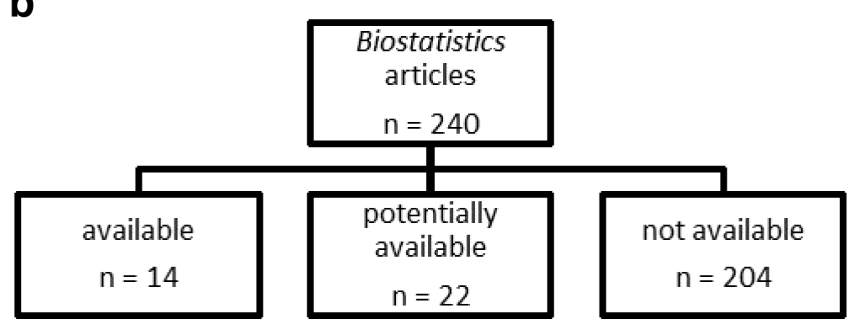

Figure 2. a: Flow charts of code availability. Randomly selected Biostatistics articles from 2006 to 2013, b: Flow charts of code availability. Randomly selected Statistics in Medicine articles from 2006 to 2013. 
A

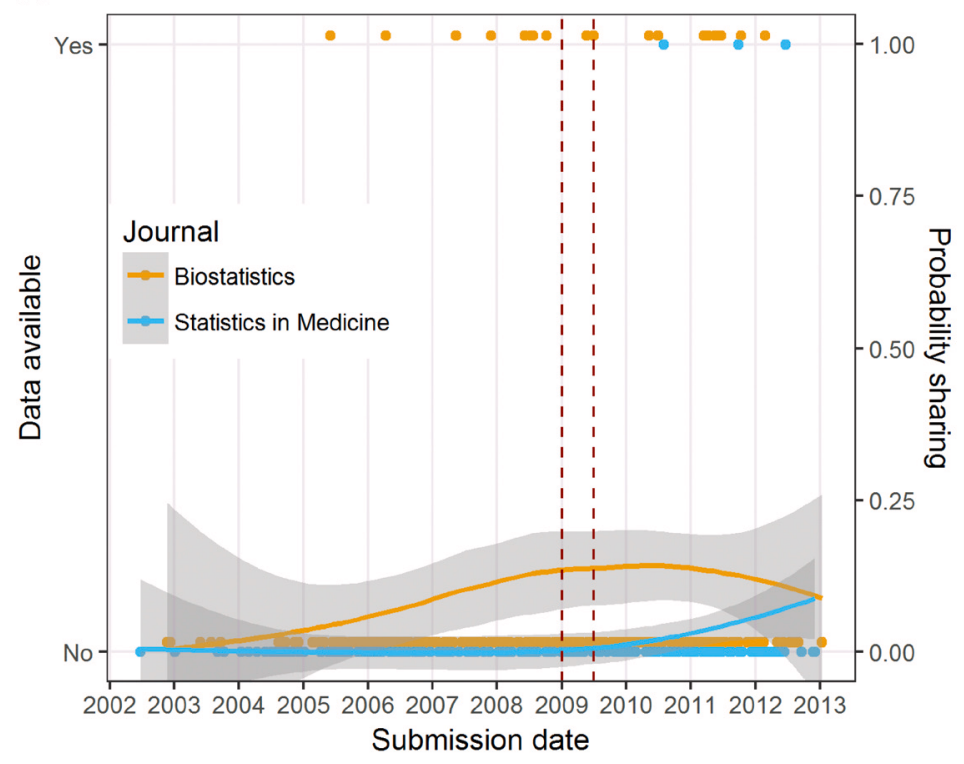

B

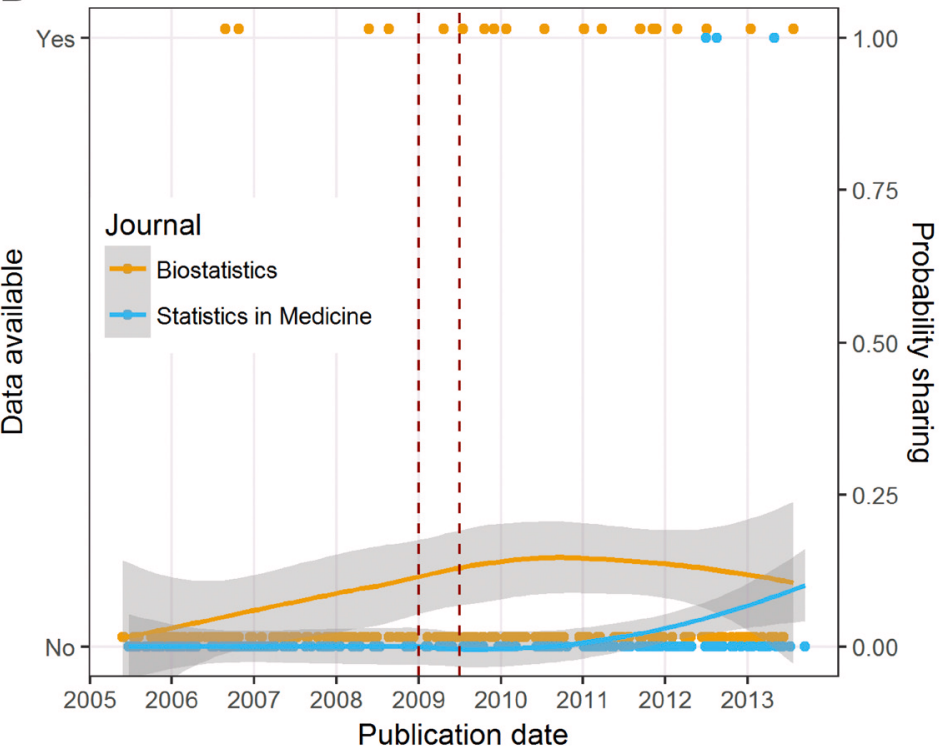

Figure 3. a: Plot of data availability over time by submission date. The dots at 'No' or 'Yes' are individual articles and the lines are a smoothed mean using a LOESS together with 95\% confidence intervals (grey areas). The red lines indicate the interim period: 1 January 2009 to 1 July 2009. b: Plot of data availability over time by publication date. The dots at 'No' or 'Yes' are individual articles and the lines are a smoothed mean using a LOESS together with 95\% confidence intervals (grey areas). The red lines indicate the interim period: 1 January 2009 to 1 July 2009.

in Figure $3 \mathrm{~b}$ are similar to those in Figure $3 \mathrm{a}$ and show that data availability and probability of sharing were increasing at Biostatistics before badges were introduced.

The code availability and probability of sharing by submission date together with a smooth curve and $95 \%$ confidence intervals are in Figures 4a. The smooth curves for Biostatistics and Statistics in Medicine are mostly on top of each other in this graph, except for a drop-off in sharing at Biostatistics in later years. This indicates no great difference in code sharing at these journals. Figure $4 \mathrm{~b}$ shows the results of the sensitivity analysis, where publication date was used instead of submission date. In this graph (Figure 4b), the smooth curve for Biostatistics and Statistics in Medicine are again mostly on top of each other, showing an increase in code sharing over time at both journals, but around mid-2011 the two curves diverged, with Statistics in Medicine showing an increase in code sharing and Biostatistics a drop.

\section{Data sharing}

The logistic regression model estimated that the probability of data sharing increased by 5.1 (95\% CI for prevalence 

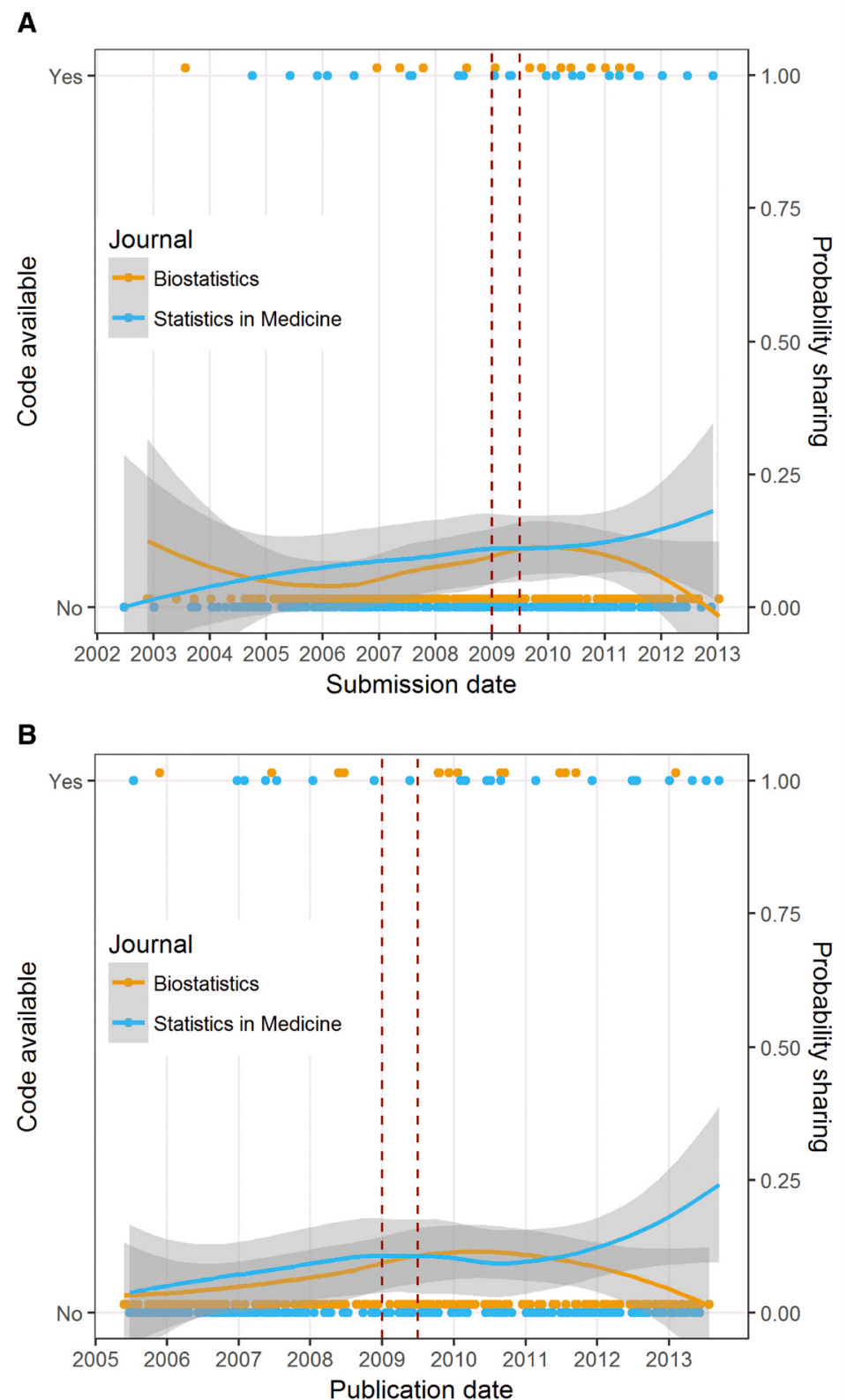

Figure 4. a: Plot of code sharing over time. The dots at 'No' or 'Yes' are individual articles and the lines are a smoothed mean using a LOESS together with 95\% confidence intervals (grey areas). The red lines indicate the interim period: 1 January 2009 to 1 July 2009 . b: Plot of code sharing over time. The dots at 'No' or 'Yes' are individual articles and the lines are a smoothed mean using a LOESS together with 95\% confidence intervals (grey areas). The red lines indicate the interim period: 1 January 2009 to 1 July 2009.

ratio: 0.6 to 14.8 , p-value: 0.942 ) times that of the control period in the interim period of 1 January 2009 to 1 July 2009. This Bayesian p-value gives an estimated $94.2 \%$ probability that the mean rate of sharing increased. After the interim period, the probability of data sharing increased by an estimated $3.5(95 \%$ CI: 1.4 to 7.4 , p-value: 0.996 ) times after badges were introduced. On an absolute scale, this difference was only a $7.3 \%$ increase in data sharing (95\% CI: 2 to 14\%). After controlling for time, badges increased the probability of data sharing at the journal by an estimated 3.9 times (95\% CI: 1.2 to 9.5 , p-value: 0.991 ).
This is comparable to the prevalence ratio of 3.5 when time was not added as a random effect, which shows that controlling for time only slightly increased the effect badges had on the probability of data sharing.

\section{Code sharing}

During the interim period, badges did not have an effect on code sharing (prevalence ratio of approximately 1). After the interim period there was an estimated $0.6 \%$ increase $(95 \%$ CI: -5 to $8 \%$, p-value: 0.549$)$ in sharing. After adjusting for time, this absolute 
difference reduced to $-1.4 \%$ (95\% CI: -7 to $5 \%$, p-value: 0.286 ). This suggests that badges did not have an impact on the probability of sharing code.

\section{Broken links}

We often encountered issues with broken hyperlinks at both journals. Forty-nine out of $76(64 \%)$ articles that provided links to data and code at Biostatistics had broken links and at Statistics in Medicine, 21 out of $50(42 \%)$ articles that provided links to data and code had broken links.

\section{Discussion}

\section{Are badges effective incentives for reproducibility?}

The results of this observational study and those of the related Kidwell et al. badge study $^{8}$ cannot accurately deduce the effectiveness of badges because of the biases of the non-randomised study design. The Kidwell et al. 2016 badge study received criticism from Hilda Bastian on its study design, analyses, and claims ${ }^{16}$. One of the criticisms was that the badges scheme was not the only intervention offered at the journal, there were four other co-interventions offered in 2014, and so any effect could not be attributed to badges alone ${ }^{16}$. Bastian reasonably argued that to isolate the impact of badges, groups that had the same conditions except badges were needed ${ }^{16}$. Our study is also exposed to similar limitations with regard to confounding as other changes may have occurred that we were not aware of. However, we can derive some insight into the effect badges had on data and code sharing from the results of both observational studies.

After the introduction of badges at Biostatistics, the probability of data sharing increased 3.5 times. This prevalence ratio might seem like a large increase but on an absolute scale it is only a $7.3 \%$ increase in the rate of data sharing, which is much lower than the $37.9 \%$ effect of badges at Psychological Science ${ }^{8}$. The large difference between the effect of badges at Biostatistics and Psychological Science could be related to differences in the culture of sharing between the two fields, and the timeframes of the studies: 2006 to 2013 for our study, versus 2012 to 2015 for Kidwell et al. Our study analysed incentives for data and code sharing at an earlier time when the reproducibility crisis was not yet a testified reality, hence researchers may have been more primed to change behaviour in the Kidwell et al. study. Also, since statisticians typically re-analyse existing datasets, it might be harder for them to share the data as they might not have the rights. This is contrary to research in psychological science where original data is normally collected and analysed, making sharing a potentially simpler task.

There was an apparent increase in data sharing before badges were introduced at Biostatistics (Figure 3a). One possibility is that articles that were submitted before the policy change could still have experienced the policy because of the time needed for peer review and resubmission. We used submission date to determine if articles were prepared before or after the policy change because we know that sharing data often takes preparation time and we believed that authors were therefore more likely to react to the policy when they were writing their first draft. However, data sharing seemed to be increasing before badges were introduced even when we used publication date in a sensitivity analysis. The reproducibility policy at Biostatistics was built on the existing framework that "allowed and encouraged authors to place supplementary materials online". Such an option of depositing supplementary material could have contributed to the rise in data sharing before badges. Also, Roger Peng assumed the role as the Associate Editor for reproducibility at Biostatistics in 2006, which might have catalysed a change in the culture of reproducibility at the journal.

Badges did not appear to have an effect on code sharing as the prevalence ratio was close to 1 with a $95 \%$ credible interval that included 1. This is an unexpected outcome as code is of great importance in the field of biostatistics. A possible explanation behind the lack of badge effect on code sharing could be our definition of code sharing, which might seem traditional compared to the reproducibility policy at Biostatistics. We defined code sharing as the availability of the code used to analyse the data (original or simulated) in the article. The policy at Biostatistics included referencing "...software that is widely available from central repositories (e.g. CRAN, Statlib)”. It is true that providing a link to a third-party repository where software packages are deposited, such as vignettes, typically contain some general code, but it often takes specialized skills to work out the code at these repositories, and they might not always explain the analyses covered in the actual published article. This is in line with what Stodden et al. recommended in their piece on reproducibility in Science, "Data and code underlying discoveries must be discoverable from the related publication, accessible, and reuseable" ${ }^{17}$.

The effect of badges on data and code sharing could have been higher if we did not encounter issues with broken links. For Biostatistics the "Supplementary Material" link did not work for the majority of articles. The current editors of Biostatistics indicated that when the publisher (Oxford) switched to a new publishing platform in January 2017, some of the supplemental material was lost in the transfer (personal communication, J Leek, 8 November 2017). Our results could have looked different if we had started collecting data in 2006, as the links could have been working back then.

Badges have been promoted as a simple solution because they are low cost. However, while collecting data for our study, we noticed that articles did not always appear to be allocated with badges correctly, implying that assigning badges is not always clear cut and journal staff may need to spend more time on verification. An alternative approach is that peer-reviewers check for data and code availability and assign badges as part of the standard peer review process. It could be that peer-reviewers prefer to have access to the data and code in order to review the 
article anyway, so this model might work, but it still requires additional time and effort on their part and as they receive little recognition for their work, plus it might be unfair to expect all peer-reviewers to check for data and code sharing.

\section{Conclusion}

Efforts are underway by the global meta-research community to strengthen the reliability of the scientific method ${ }^{18}$. Data and code sharing is an indispensable part of the movement towards science that is open; where scientific truth is not a questionable commodity, but is easily accessible, replicable, and verifiable ${ }^{19}$. The cultural shift towards reproducible science is complex and it calls for a twofold change in the attitudes of individual researchers toward reproducibility, and the leadership provided by the systems and services that support scientific research. As such, journals, universities, government bodies, and funders are key players in promoting this culture. Transparency and reproducibility are elements central to strengthening the scientific method, and data and code provide the key to scientific truth ${ }^{12}$. As Peng argued in Science, the culture of reproducibility will not drastically change overnight, but simply bringing the notion of reproducibility to the fore and making it routine will make a difference ${ }^{3}$. Badges are already being used by journals including Biostatistics, Psychological Science, British Medical Journal Open Science, and Association for Computing Machinery to encourage researchers to share the evidence behind their work $^{1,20}$. Based on this observational study and a previous study, it appears that badges do help to increase data sharing, but a randomised trial is needed to better estimate their true effect, as well as studies of the additional time needed to implement and maintain them.
Data availability

Anonymised data and the code used in the analyses are publicly available at: https://doi.org/10.6084/m9.figshare. 5687548

\section{Consent}

An ethics exemption was granted by the Office of Research Ethics and Integrity at the Queensland University of Technology for this study (exemption number: 1700001051). No consent was needed as all data collected and analysed in this study were publicly available.

\section{Competing interests}

No competing interests were disclosed

\section{Grant information}

This study was supported in kind by the Institute of Health and Biomedical Innovation at the Queensland University of Technology.

The funders had no role in study design, data collection and analysis, decision to publish, or preparation of the manuscript.

\section{Acknowledgements}

Thanks to one of the current Editors of Biostatistics, Professor Jeffrey Leek, for his prompt response to our enquiry. Sincere thanks goes to my $\mathrm{PhD}$ colleague, Victoria McCreanor, for reading through the draft manuscript and providing feedback.
1. Peng RD: Reproducible research and Biostatistics. Biostatistics. 2009; 10(3): 405-408.

PubMed Abstract | Publisher Full Text

2. Goodman SN, Fanelli D, loannidis JP: What does research reproducibility mean? Sci Transl Med. 2016; 8(341): 341ps12. PubMed Abstract | Publisher Full Text

3. Peng RD: Reproducible research in computational science. Science. 2011; 334(6060): 1226-1227.

PubMed Abstract | Publisher Full Text | Free Full Tex

4. National Academy of Sciences, National Academy of Engineering (US) and Institute of Medicine (US) Committee on Science, Engineering, and Public Policy: On Being a Scientist: A Guide to Responsible Conduct in Research: Third Edition. Washington, DC: The National Academies Press (US); 2009. PubMed Abstract | Publisher Full Text

5. Rowhani-Farid A, Barnett AG: Has open data arrived at the British Medical Journal (BMJ)? An observational study. BMJ Open. 2016; 6(10) Publisher Full Text

6. Ioannidis JP, Khoury MJ: Assessing value in biomedical research: The PQRST of appraisal and reward. JAMA. 2014; 312(5): 483-484. PubMed Abstract | Publisher Full Text | Free Full Text

7. Rowhani-Farid A, Allen M, Barnett AG: What incentives increase data sharing in health and medical research? A systematic review. Research Integrity and Peer Review. 2017; 2(1): 4 Publisher Full Text

8. Kidwell MC, Lazarević LB, Baranski E, et al:: Badges to Acknowledge Open Practices: A Simple, Low-Cost, Effective Method for Increasing Transparency.
PLoS Biol. 2016; 14(5): e1002456

PubMed Abstract | Publisher Full Text | Free Full Text

9. 1,500 scientists lift the lid on reproducibility: Nature News \& Comment.

10. Ebersole CR, Axt JR, Nosek BA: Scientists' Reputations Are Based on Getting It Right, Not Being Right. PLOS Biol. 2016; 14(5): e1002460. PubMed Abstract | Publisher Full Text | Free Full Text

11. Barbui $C$ : Sharing all types of clinical data and harmonizing journal standards. BMC Med. 2016; 14(1): 63. PubMed Abstract | Publisher Full Text | Free Full Text

12. Iqbal SA, Wallach JD, Khoury MJ, et al.: Reproducible Research Practices and Transparency across the Biomedical Literature. PLOS Biol. 2016; 14(1): e1002333.

PubMed Abstract | Publisher Full Text | Free Full Text

13. van Panhuis WG, Paul $P$, Emerson $C$, et al.: A systematic review of barriers to data sharing in public health. BMC public health. 2014; 14: 1144 PubMed Abstract | Publisher Full Text | Free Full Text

14. Deddens JA, Petersen MR: Approaches for estimating prevalence ratios. Occup Environ Med. 2008; 65(7): 501-506. PubMed Abstract | Publisher Full Text

15. Vines TH, Albert AY, Andrew RL, et al.: The Availability of Research Data Declines Rapidly with Article Age. Curr Biol. 2014; 24(1): 94-97. PubMed Abstract | Publisher Full Text

16. Bastian H: Bias in Open Science Advocacy: The Case of Article Badges for Data Sharing. In., PLOS Blogs; 2017; 2017. Reference Source

17. Stodden V, McNutt M, Bailey D, et al.: Enhancing reproducibility for 
computational methods. Science. 2016; 354(6317): 1240-1241. PubMed Abstract | Publisher Full Text

18. Ioannidis JP, Fanelli D, Dunne DD, et al.: Meta-research: Evaluation and Improvement of Research Methods and Practices. PLOS Biol. 2015; 13(10): e1002264.

PubMed Abstract | Publisher Full Text | Free Full Text
19. Reproducibility and reliability of biomedical research: improving research practice. In.: The Academy of Medical Sciences. 2015. Reference Source

20. Sena ES: Inaugural editorial: advancing preclinical and translational research of relevance to medicine. BMJ Open Science. 2017; 1(1). Publisher Full Text 


\title{
Open Peer Review
}

\section{Current Peer Review Status:}

\section{Version 1}

Reviewer Report 12 February 2018

https://doi.org/10.5256/f1000research.14635.r30239

(C) 2018 Greshake B. This is an open access peer review report distributed under the terms of the Creative Commons Attribution License, which permits unrestricted use, distribution, and reproduction in any medium, provided the original work is properly cited.

\author{
Bastian Greshake \\ 1 Division of Environmental Genomics and Systems Biology, Lawrence Berkeley National \\ Laboratory, Berkeley, CA, USA \\ 2 Open Humans (openhumans.org), Frankfurt am Main, Germany
}

This study evaluates the effects that badging has on availability of both data and source code. Badges that highlight and reward articles that share data/code have been described as a simple way to increase sharing behavior and thus reproducibility. This makes this study highly relevant and interesting to the field of meta-research. To measure whether badging has this desired effect the authors compare the availability of data/code in the journal Biostatistics - which introduced badges in 2009 - with a control journal, Statistics in Medicine. The main finding is that there seems to be a small effect on data sharing associated with the introduction, while badges appear to have no effect on the sharing of code.

While the statistical methods are appropriate and sound, the study has some limitations given by the data set on how the authors coped with missing data. First of all, and this is probably the main limitation, there is only a small set of publications in the authors' set of 480 that have data/code associated with them. Only around $8 \%$ of all publications have code attached, a number that drops to less than $5 \%$ for data. Given this overall low rate of sharing - and the large time span covered there is a lot of fluctuation in the observed sharing behavior for both code and data (c.f. Figure 3 \& 4) between the two journals. Given that only Statistics in Medicine is used as a control journal it is unclear how much of these differences are cultural between the audiences of the two journals (also c.f. that code sharing is more prevalent in SIM than Biostatistics and increases more despite the lack of badges). A larger set of control-journals would potentially minimize this 'culture'-effect (though requiring a potentially unreasonably large time investment).

In addition to this there are further effects that complicate a clear inference of the effect of badges:

1. There is a general trend that "Open Science" and data sharing are becoming more popular (c.f. Tenopir et al. $(2015)^{1}$ ). In line with this the authors find that the sharing of data in Biostatistics already increased prior to the introduction of badges, even when going for 
conservative measures. Additionally there is an observed increase in data sharing in Statistics in Medicine in later years, albeit from a lower baseline. I think this general trend should be included in the discussion.

2. The authors additionally find that the links for $64 \%$ of articles that provide data/code are broken in Biostatistics and highlight the issue that OUP had with losing (not only) supplementary data. The authors treat these articles as having data/code not publicly available. This not only leads to a marked decrease overall decrease of articles with data/code available but can furthermore be a potential source of bias. For OUPs Molecular Biology and Evolution it was seen that these losses on behalf of OUP not only affected supplementary materials but even individual years worth of full text articles (c.f. http://rossmounce.co.uk/2017/04/02/open-letter-to-oxford-university-press/). If OUP also lost the supplementary materials of certain publication date ranges for Biostatistics this will heavily bias the analysis presented here.

Given these limitations I would be cautious to assume whether badges have a positive effect or not on data availability at this point.

To improve the resolution by adding more data and to decrease the biases mentioned in 2) I recommend taking the original data/code availability statements at face value and not treat broken links as publications without data/code. I think it is defendable to assume that the data/code was available at the time of publication when the main text suggests so. Doing this the potential effect of badges should become more pronounced as it is not hidden by the 'link-rot' that OUP is responsible for.

Overall, I think this is a valuable contribution to the field of meta-research and our understanding of incentives around sharing and reproducibility. I hope that the minor improvements suggested above can be useful to make this data set even more useful.

\section{References}

1. Tenopir C, Dalton E, Allard S, Frame M, et al.: Changes in Data Sharing and Data Reuse Practices and Perceptions among Scientists Worldwide. PLOS ONE. 2015; 10 (8). Publisher Full Text

\section{Is the work clearly and accurately presented and does it cite the current literature?} Yes

Is the study design appropriate and is the work technically sound? Partly

Are sufficient details of methods and analysis provided to allow replication by others? Yes

If applicable, is the statistical analysis and its interpretation appropriate? Partly

Are all the source data underlying the results available to ensure full reproducibility? Yes 


\section{Are the conclusions drawn adequately supported by the results?}

Partly

Competing Interests: No competing interests were disclosed.

Reviewer Expertise: Bioinformatics, meta-science

I confirm that I have read this submission and believe that I have an appropriate level of expertise to confirm that it is of an acceptable scientific standard, however I have significant reservations, as outlined above.

\section{Author Response 01 Mar 2018}

\section{Anisa Rowhani-Farid}

We looked for additional control journals to minimise the 'culture'-effect described above. The journals that met the eligibility criteria of being in the field of applied statistics and/or biostatistics and which had a similar relative high prestige in the field as per Biostatistics were the following:

\section{Statistical Methods in Medical Research}

Biometric Journal

Biometrics

Royal Statistical Society - Series C Applied Statistics

However, none of these journals were suitable as controls as they all had reproducibility policy changes during the time frame of our study (2006 to 2013) or had a reproducibility policy in place before that timeframe. We have added an explanation of this in our methods.

The general trend towards more open science is now discussed in the Discussion. We used sensitivity analyses that adjusted for the effects of time (including trends), and the effects of badges were relatively similar to the unadjusted results.

We have conducted sensitivity analyses for data and code sharing to account for the broken links at the journal. These results have been added and discussed in the article.

Competing Interests: No competing interests 
(C) 2018 Nilsonne G. This is an open access peer review report distributed under the terms of the Creative Commons Attribution License, which permits unrestricted use, distribution, and reproduction in any medium, provided the original work is properly cited.

\section{Gustav Nilsonne}

1 Stress Research Institute, Stockholm University, Stockholm, Sweden

2 Department of Clinical Neuroscience, Karolinska Institutet, Stockholm, Sweden

This study is an observational investigation of the effect of badges/kitemarks on sharing of data and code. The authors compared one journal that introduced badges in 2009 (Biostatistics) to one that did not (Statistics in Medicine). The main finding was that badges were associated with an increase in open data publication, although with a considerably smaller effect than that observed in the one previous investigation of this question. The finding is important and may influence publishing policies.

The main limitation of this study is that only two journals were included. The authors provide a strong reason for this, namely that Biostatistics is unique in having introduced badges sufficiently long ago to have a period of follow-up of several years.

Statistical methods are sound; descriptive statistics are very clear and inferential statistics are appropriate. The choice to use the six months before badges came into effect (the "interim period") as a reference period is arbitrary, and it is not possible for a reader to assess whether six months would capture most papers undergoing review and revision at the time the policy was introduced. If papers took longer, that could contribute to the increasing rates of data sharing observed in the run-up to the introduction of badges, seen in figure 3. Thus, the choice of reference period is likely to yield a conservative estimate of the effect of the policy.

Papers were coded by one person only, and that is a minor weakness. My experience with coding articles for open data and similar outcomes leads me to think that the main reason to have two coders in this context is to reduce the risk of error, rather than to handle interrater differences in assessments. It is not likely, in my opinion, that errors in the coding have effects on a magnitude approaching that seen for the intervention.

I have cursorily reviewed the data and analysis code also. The data variable names are selfexplanatory. The code, written in Markdown, is sufficiently documented with inline headings. However, the code requires some modification before it can be run independently, and this is not documented. For example, on line 18, the code attempts to load the data, by calling a file that is not provided. The data must instead be loaded by opening the provided R workspace file. I suggest that the data be made available in a csv or txt file, which is safer for accessibility in the long term and across softwares. The code could then call this file.

The ancillary finding of high rates of broken hyperlinks to data at both journals is interesting, as is the explanation that supplementary data were lost by one of the journals during a platform migration. I have several times advanced the argument that this risk is one motivation for publishing data and code using dedicated repositories, but I have not previously seen an empirical observation such as this one. I suggest that this finding should be mentioned in the abstract. 
One minor detail: In figure 4, the legend does not explain the difference between panels $a$ and $b$, as in figure 3.

In summary, this article makes an important contribution towards our understanding of effects of badges on publication of open data and open code. I am happy to approve this article.

Is the work clearly and accurately presented and does it cite the current literature? Yes

Is the study design appropriate and is the work technically sound?

Yes

Are sufficient details of methods and analysis provided to allow replication by others? Yes

If applicable, is the statistical analysis and its interpretation appropriate? Yes

Are all the source data underlying the results available to ensure full reproducibility? Yes

Are the conclusions drawn adequately supported by the results? Yes

Competing Interests: I am the former chair of the Open Science Badges Committee.

Reviewer Expertise: Neuroscience, Meta-science

I confirm that I have read this submission and believe that I have an appropriate level of expertise to confirm that it is of an acceptable scientific standard.

Author Response 01 Mar 2018

Anisa Rowhani-Farid

We have updated the Rmarkdown code and added an R data set so anyone can independently run the analysis.

Although ARF coded all the data, AGB verified 20 observations and the two authors debated a handful of cases where it was not clear. In general the sharing of data or not was relatively clear-cut and hence we believe that one coder should be sufficient.

We agree that the six month date window prior to the introduction of badges is arbitrary, and this is why we also plotted the sharing rates over time so that readers could see when any changes in sharing took place, and why we tried the alternative time scale of submission date. 
We have now mentioned broken links in in the abstract.

We have corrected this: "One minor detail: In figure 4, the legend does not explain the difference between panels a and b, as in figure 3".

Competing Interests: No competing interests.

The benefits of publishing with F1000Research:

- Your article is published within days, with no editorial bias

- You can publish traditional articles, null/negative results, case reports, data notes and more

- The peer review process is transparent and collaborative

- Your article is indexed in PubMed after passing peer review

- Dedicated customer support at every stage

For pre-submission enquiries, contact research@f1000.com 